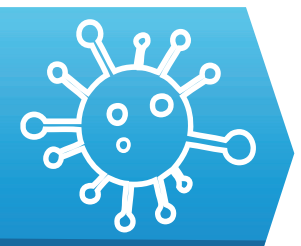

INFECTIOUS DISEASESS

Internal Medicine Department, Pt. BD Sharma PGIMS, Rohtak, India

\section{Scrub typhus infection, not a benign disease: an experience from a tertiary care center in Northern India}

\author{
Deepak Jain, Nitya Nand, Kajaree Giri, Jaikrit Bhutani
}

\begin{abstract}
Introduction. Scrub typhus is an acute febrile illness caused by Orientia tsutsugamushi. It is characterised by fever, rash, myalgia and diffuse lymphadenopathy. Most common complications are sepsis, shock, acute respiratory distress syndrome (ARDS), hepatitis, pre renal azotemia and multi organ dysfunction syndrome (MODS). Mortality rates range from $7-30 \%$ in untreated cases. Scrub typhus is endemic to a part of the world known as 'the tsutsugamushi triangle'. River banks, grassy areas generally harbour scrub typhus infection; however, recently there has been an increase in prevalence of infection from dry regions like Haryana.
\end{abstract}

Objective. To assess the clinical spectrum and complications of scrub typhus infection at a tertiary care centre in North India.

Material and methods. An observational study was conducted on patients $>14$ years old with acute febrile illness $>7$ days duration, admitted indoor from emergency department, from July to November 2017. Suspected cases were tested for specific IgM antibodies against Orientia tsutsugamushi by ELISA.

Results. Among the 230 patients, screened for scrub typhus infection, 39 (16.95\%) came out to be positive. Most common patient complaints were fever followed by cough and breathlessness, myalgia, nausea, vomiting and behavioral abnormality. $15 \%$ of patients required inotropic support initially and $48 \%$ had oxygen saturation of less than $90 \%$ at the time of presentation. Pleural effusion and crepitations were present in $41 \%$ of patients. Most common biochemical alterations were: abnormal liver function tests $(95 \%)$, followed by thrombocytopenia, anemia, abnormal renal function tests, and hyponatremia. 12 patients $(30.7 \%)$ were shifted to intensive care unit, 8 of which $(20.5 \%)$ needed invasive mechanical ventilation and 4 patients $(10.3 \%)$ underwent hemodialysis. Various complications were noted in $89.7 \%$ of cases, the most common being ARDS followed by sepsis, acute kidney injury (AKI) and meningitis. The mortality rate in this study was $18 \%$.

Conclusions. This study emphasizes that scrub typhus infection is on a rampant resurgence and it is associated with significant complications. High degree of suspicion as well as development of effective measures to treat, control and prevent is critical to lower the disease burden.

Keywords: scrub typhus, ARDS, sepsis, AKI

\section{Introduction}

Scrub typhus is a bacterial zoonosis caused by Orientia tsutsugamushi presenting as acute febrile illness with variable severity. An estimated one billion people are at risk for scrub typhus and one million cases occur worldwide, annually [1]. It is characterized by fever, an exanthematous rash, myalgia and diffuse lymphadenopathy. Most common complications are sepsis, shock, acute respiratory distress syndrome (ARDS), hepatitis, pre renal azotemia and multiorgan dysfunction syndrome (MODS). Mortality rate ranges from $7-30 \%$ in untreated cases [2-5].

Scrub typhus is endemic to a part of the world known as 'the tsutsugamushi triangle', which extends from the Northern Japan and far East Russia in the North, to 
Northern Australia in the South and to Pakistan in the West. India is an integral component of this triangle. In India the disease is widely spread in the Sub-Himalayan belt extending from Jammu and Kashmir to Nagaland and also found in West Bengal, Tamil Nadu and Kerala. However, in recent times frequent outbreaks of this disease has been witnessed in many parts of the country: Uttarakhand, Kumaon, Uttarpradesh, Rajasthan, Haryana in the North, Meghalaya in the North-East, Pondicherry, Andhra Pradesh, Kerala, Tamil Nadu in the South and Goa in the West. This has led to a revision of the assumption that scrub typhus is limited to the Sub-Himalayan belt only [6-9].

Haryana is a North Indian state with dry weather and scanty rainfall where scrub typhus is usually not considered a differential in the diagnosis of acute febrile illness presenting with thrombocytopenia or MODS. However, recently there has been an increase in the prevalence of scrub typhus cases reported from this region, which can be attributed mostly to the occupation of the inhabitants (farmers/agricultural workers) and their exposure to bushes, woods, environmental animals and rodents [1]. High burden of complications have been documented in this infection and the case fatality rate can be up to $30-70 \%$ if no appropriate treatment is received. A high degree of suspicion for early detection of the disease and development of effective measures to treat, control and prevent the same is a critical public health issue [10].

Hence, this present study was undertaken in view of an increased incidence of acute febrile illness in Northern India, associated with thrombocytopenia and multi-organ failure, to assess the burden of scrub typhus along with its clinical spectrum, complications and outcomes.

\section{Material and methods}

A longitudinal, observational study was conducted between July 2017 - November 2017 in a tertiary care center in North India. Prior approval of the study protocol from Institutional Ethics Committee (IEC) was obtained. All patients over 14 years of age with acute undifferentiated febrile illness of more than 7 days duration attending the medicine emergency department and later admitted in medicine inpatient wards were screened. An informed and written consent was taken from every patient before inclusion in the study. Detailed history regarding occupation and area of residence, clinical examination including lymphadenopathy, pleural effusion, hepato-splenomegaly were done and also a careful search for eschars was performed in every patient. Examination included basic laboratory work up such as complete blood count, renal function tests, liver function tests including serum bilirubin (direct and indirect), aspartate aminotransferase (AST/ SGOT), alanine aminotransferase (ALT/SGPT), alkaline phosphatase (ALP) and serum albumin. All patients were evaluated for other endemic febrile diseases like malariamalaria rapid diagnostic card test $\left(\right.$ CareStart $^{\mathrm{TM}}$ malaria
HRP2/pLDH (Pf) by Access Bio, Inc.) and peripheral blood film, enteric fever - Widal test and typhidot test ( it consists of a dot ELISA kit that detects IgM and IgG antibodies against the outer membrane protein of Salmonella typhi), dengue- dengue Ns1 antigen and $\operatorname{IgM}, \operatorname{IgG}$ antibody card tests (Zephyr Biomedicals, India) and leptospirosis - indirect IgM leptospira antibody test by ELISA. Chest roentgenograms and electrocardiograms were done for every patient. Other investigations including blood cultures, cerebrospinal fluid (CSF) analysis, ultrasonography of abdomen and magnetic resonance imaging (MRI) of brain were performed as indicated. In CSF examination apart from routine biochemistry, adenosine deaminase (ADA) levels and polymerase chain reaction (PCR) tests were done to rule out Mycobacterium tuberculosis. Scrub typhus was diagnosed by serology testing for IgM antibodies against Orientia tsutsugamushi using commercial enzyme linked immunosorbent assay (ELISA) kits. The kit uses a specific $56 \mathrm{kDa}$ recombinant antigen of Orientia tsutsugamushi and has a sensitivity and specificity of $>90 \%$ when compared with other gold standard tests namely immunofluorescent antibody (IFA) test, indirect immune peroxidase test or agglutination test (Weil Felix test). The kits were procured from National Centre for Disease Control (NCDC), New Delhi (India), which is a WHO approved central government institute for conducting these tests.

Any patient with acute febrile illness, having clinical and biochemical features suggestive of scrub typhus, tested negative for other common diseases prevalent in the study area (dengue, leptospirosis, malaria and enteric fever) and positive for IgM ELISA scrub typhus was considered as confirmed cases. The various systemic complications (acute kidney injury, acute hepatitis, acute respiratory distress syndrome (ARDS), pneumonitis, multiple organ dysfunction syndrome (MODS), meningitis, septic shock, disseminated intravascular coagulation (DIC), and pancreatitis) in the patients with scrub typhus were defined as per standard textbook definitions [8].

Confirmed cases of scrub typhus were given doxycycline $200 \mathrm{mg}$ in two divided doses for 14 days. Appropriate organ support measures like inotropic support, mechanical ventilation, renal replacement therapy and blood and blood products were provided as per patients' requirements.

Socio-demographics, clinical signs/symptoms, clinical outcomes are recorded in a pre-designed structured proforma. Data are analyzed using SPSSv16 software. Results are presented as frequency and percentages for qualitative variables and are represented in graphs and tables.

\section{Results}

Among the 230 patients who were screened, 44 (19\%) patients were diagnosed to have scrub typhus positive, but 5 patients were excluded from the study owing to concomitant dengue and malaria infection. Hence 39 
cases $(16.95 \%)$ fulfilled the criteria of confirmed cases and were included in the study.

Twenty-four cases $(61.53 \%)$ were reported during the months of September - October. The mean age of the patients was 38 years. Among the 39 purely scrub typhus positive cases, $24(61 \%)$ patients were females. In the present study, $12(30.76 \%)$ patients were involved in farming and agricultural works outdoors, 15 (38.48\%) patients were labourers and the rest (30.76\%) were homemakers.

All the patients included in the study presented with complaints of fever, the mean duration of which was 10.2 days (before hospitalisation). It was followed by cough and breathlessness, myalgia, nausea,vomiting, behavioural abnormality, abdominal pain, headache and bleeding tendencies (gums and oral cavity). The clinical spectrum of the patients is depicted in figure 1. Rashes were found in 17 patients (43.6\%) predominantly in the lower limbs, back side of the trunk and anterior chest wall during the course of the illness. Eschar was noted in 5 patients $(12.8 \%)$ in inframammary folds, axillary folds, anterior chest wall and legs.

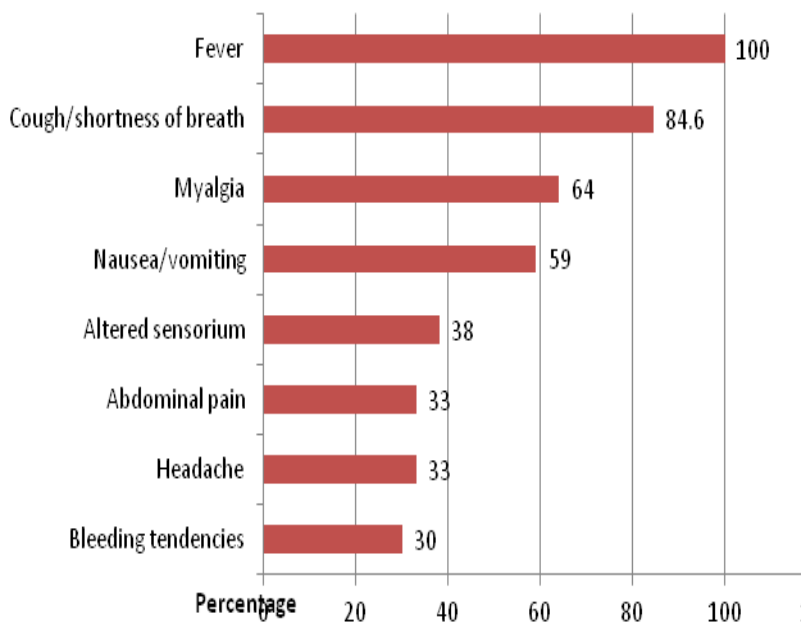

Figure 1. Clinical symptoms among hospitalized patients with scrub typhus $(\mathrm{n}=39)$.

Mean systolic blood pressure at presentation was $105 \mathrm{mmHg}$ and 8 patients presented with systolic blood pressure (SBP) less than $90 \mathrm{mmHg}$, of whom 6 patients $(15 \%)$ required inotropic support. The mean oxygen saturation of patients on the day of presentation was $88 \%$. 19 patients (48\%) had oxygen saturation less than $90 \%$ in room air whereas $10 \%$ had oxygen saturation less than $80 \%$ in room air. The mean urine output of the patients on the day of presentation was $683.59 \mathrm{ml} /$ day. On chest auscultation, rhonchi and crepitations were present in 16 (41\%) patients during presentation; however isolated crepitations were noted only in $8(20.5 \%)$ patients. Pleural effusion was suspected clinically and later documented by ultrasonography in $16(41 \%)$ patients also. Hepatomegaly (46\%) and splenomegaly (51\%) were also noted. 16 patients (41\%) also had jaundice. 28 patients $(71 \%)$ had urine output less than $1000 \mathrm{ml} /$ day on the day of admission in hospital. Generalized lymphadenopathy was noted in 8 $(20.5 \%)$ patients. The clinical signs among the hospitalized patients are summarized in table I.

Table I. Clinical signs among hospitalized patients with scrub typhus $(\mathrm{n}=39)$.

\begin{tabular}{l|c} 
Parameters & $\mathrm{n}(\%)$ \\
Rashes & $17(43.6)$ \\
Eschar & $5(12.82)$ \\
$\mathrm{SBP}<90 \mathrm{mmHg}$ at presentation & $8(20.5)$ \\
$\mathrm{SpO} 2$ at presentation & \\
$<80 \%$ & $4(10)$ \\
$80-90 \%$ & $15(38)$ \\
$>90 \%$ & $20(52)$ \\
Generalized lymphadenopathy & $8(20.5)$ \\
Crackles and rhonchi & $16(41)$ \\
Pleural effusion & $16(41)$ \\
Jaundice & $16(41)$ \\
Hepatomegaly/splenomegaly & $18 / 20(46 / 51)$ \\
Oliguria & \\
$<500 \mathrm{ml} /$ day & $13(33)$ \\
$500-1000 \mathrm{ml} / \mathrm{d}$ & $15(38)$ \\
$>1000 \mathrm{ml} /$ day & $11(28)$ \\
SBP $=$ systolic blood pressure &
\end{tabular}

The biochemical profile of the patients are summarized in table II. Most common biochemical alterations were abnormal liver function tests followed by thrombocytopenia of less than $100000 / \mathrm{cmm}$ and anemia. Raised blood urea levels (more than $45 \mathrm{mg} / \mathrm{dl}$ ) was observed in 22 patients and serum creatinine more than $1.5 \mathrm{mg} / \mathrm{dl}$ was noted in 10 patients. The most common electrolyte disturbance prevalent was hyponatremia observed in $46 \%$ of patients. Leucopenia (TLC count less than $4000 / \mathrm{cmm}$ ) was seen in $16(41 \%)$ patients and leucocytosis (TLC count more than $12000 / \mathrm{cmm})$ was evident in $8(20.5 \%)$ patients. Proteinuria was observed in $28(72 \%)$ patients and 22 patients $(56 \%)$ had metabolic acidosis. Mean serum protein level of the patients was $5.2 \mathrm{~g} / \mathrm{dl}$. Incidentally, some unique observations were made in the biochemical parameters. AST values were higher than ALT values in $70 \%$ of cases. Alkaline phosphatase was elevated in all 39 patients included in the study. However, ultrasound examination revealed normal echo texture of liver without any features of biliary duct obstruction. Chest roentgenograms revealed bilateral pulmonary infiltrates in 16 patients $(41 \%)$ ). On CSF examination, mean CSF protein, glucose and ADA levels were $150.16 \mathrm{mg} / \mathrm{dl}, 53.23 \mathrm{mg} / \mathrm{dl}$ and $16.98 \mathrm{U} / \mathrm{L}$ respectively. Mean total count of CSF leucocyte and lymphocyte percentage were 54 cells $/ \mathrm{cmm}$ and $95.66 \%$ respectively. 
Table II. Biochemical profile of patients with scrub typhus ( $\mathrm{n}=39$ ).

\begin{tabular}{lcc}
\hline Parameters & $\mathrm{n}(\%)$ & Mean values \\
\hline $\begin{array}{l}\text { Anemia } \\
\text { (hemoglobin }<10 \mathrm{~g} / \mathrm{dl})\end{array}$ & $28(72)$ & 8.76 \\
$\begin{array}{l}\text { Total leucocyte count } \\
<4000 / \mathrm{cmm}\end{array}$ & & 7085 \\
$4000-12000 / \mathrm{cmm}$ & $16(41)$ & \\
$>12000 / \mathrm{cmm}$ & $15(38.5)$ & \\
Thrombocytopenia & $8(20.5)$ & 92051 \\
$<50,000 / \mathrm{cmm}$ & $18(46.2)$ & \\
$50,000-100000 / \mathrm{cmm}$ & $1128.2)$ & \\
$>100000 / \mathrm{cmm}$ & $1025.6)$ & \\
$\begin{array}{l}\text { Altered LFTs } \\
\text { ALT/AST }(\mathrm{U} / \mathrm{L})\end{array}$ & $37(98)$ & $83.36 / 128.51$ \\
ALP $(\mathrm{U} / \mathrm{L})$ & $39(100)$ & 238.51 \\
Hyponatremia & $18(46.2)$ & 135 \\
(serum Sodium $<135 \mathrm{meq} / \mathrm{L})$ & & \\
Altered RFTs & & \\
Blood urea $>45 \mathrm{mg} / \mathrm{dl}$ & $22(56.4)$ & 69.54 \\
Serum creatinine $>1.5 \mathrm{mg} / \mathrm{dl}$ & $1025.6)$ & 1.15
\end{tabular}

(LFT- liver function tests, RFT- renal function tests), ASTaspartate aminotransferase, ALT- alanine aminotransferase, ALPalkaline phosphatase

Patients were treated with doxycycline $100 \mathrm{mg}$ twice daily for 14 days. Various complications were noted in 35 (89.7\%) patients. The most common complication was ARDS $(\mathrm{n}=28,72 \%)$ followed by sepsis with MODS $(\mathrm{n}=24$, $62 \%)$, acute kidney injury (AKI) $(\mathrm{n}=22,56.4 \%)$, shock $(\mathrm{n}=8,20.5 \%)$, meningitis $(\mathrm{n}=6,15.3 \%)$ and disseminated intravascular coagulation (DIC) $(\mathrm{n}=5,13 \%)$. Meningitis was documented in 6 patients with CSF examination revealing increased proteins with lymphocytic pleocytosis. A very unusual complication of scrub typhus -perforation peritonitis was found in one young patient. 12 patients $(30.7 \%)$ were shifted to intensive care unit (ICU) in view of deteriorating health condition of whom 8 patients (20.5\%) needed invasive mechanical ventilation (mean duration of ventilation required 6.8 days) and 4 (10.3\%) patients underwent hemodialysis (mean number of sessions required 6.75). Figure 2 depicts the clinical outcomes of the patients with scrub typhus.

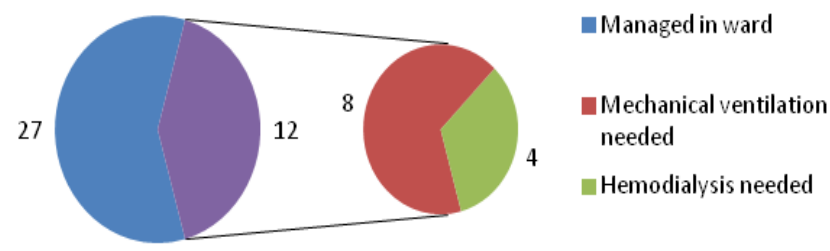

Figure 2. Clinical outcome of the patients with scrub typhus $(n=39)$.

Despite our best efforts, out of 39 patients, 7 patients succumbed to illness: 3 patients were in ARDS, 3 patients were in DIC and 1 patient was of perforation peritonitis. The mortality rate was $18 \%$ in this study. The rest of the patients recovered completely with treatment. Figure 3 depicts clinical outcomes of the patients with complications.

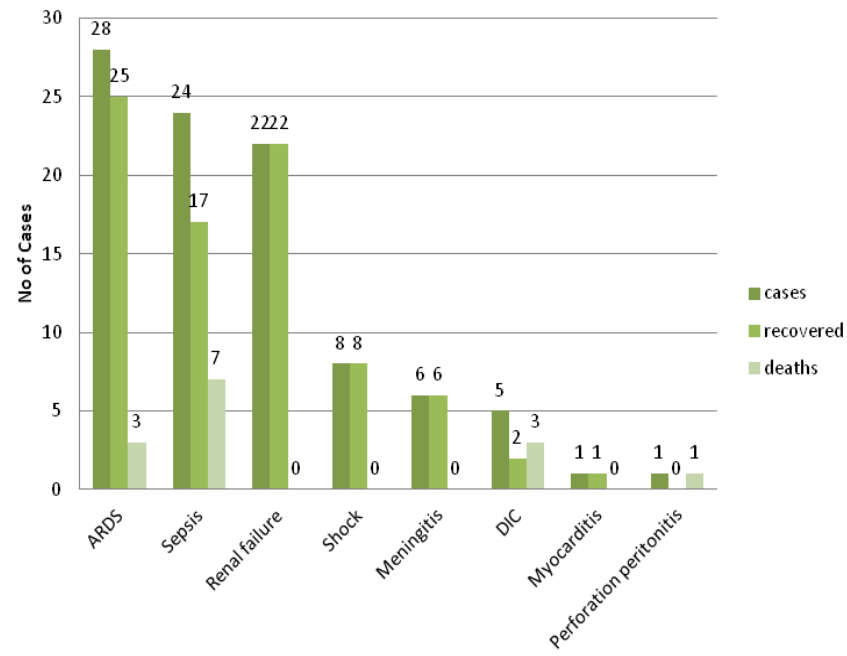

Figure 3. Clinical outcome of the patients with complications.

\section{Discussion}

Scrub typhus is caused by Orientia tsutsugamushi an obligate intracellular bacterium of the Rickettsiaceae family which is transmitted to humans by the bite of the larva (chiggers) of the trombiculid mite [8]. Infected mites are found particularly in areas like forest clearings, river banks and grassy regions during the rainy season when the mites lay eggs. The clinical spectrum of the disease often mimics complicated malaria and its prompt response to doxycycline which is often included in the antimalarial treatment, has led to underestimation of the disease prevalence despite its significant presence [1].

There has been a recent increase in prevalence of scrub typhus worldwide and in India [11]. It has been shown that scrub typhus is no longer a benign infection as previously thought. Moreover, the epidemiological pattern of the distribution of the disease has changed, as it is no longer confined only to the sub Himalayan states. This longitudinal study was an attempt to explore the complications as well as the clinical outcomes in the patient infected with scrub typhus.

Scrub typhus is a disease of monsoon and the clustering of cases are witnessed mainly in July to October months, namely in the monsoon and post monsoon seasons ('post monsoon surge'). However, some studies in South India have also reported outbreaks of scrub typhus during cooler months $[7,9]$.

Scrub typhus is a disease of hilly areas and scrub vegetations, though it has also been reported from diverse habitats such as sea shore, rice fields and semi dessert areas. Haryana being a dry state with scanty rainfall, the disease was considered less likely in the differentials of acute febrile 
illness, until lately when a study revealed recent increase in incidence of scrub typhus infection from this region [1]. Farmers, agricultural workers are the predominant population affected by this disease which is transmitted by the bite of trombiculid mites or 'chiggers'. People having exposure to bushes, piles of wood, domestic animals and rodents are at significant risk of this infection. In this study, farmers and labourers constitute the major population, an observation similar to various studies in India and abroad $[6,12]$. An increase in scrub vegetations in the monsoon and cooler months favor the growth of the vectors, infecting the farmers who are involved in the harvesting activities in the fields in that season [7]. $61 \%$ of the patients in the study cohort are female, probably due to the increased proportion of the female population in Haryana who are involved in farming and agricultural works.

The patients in our study had a wide spectrum of clinical manifestations, which is similar to various studies all over India [13-16]. Scrub typhus commonly mimics other tropical infections and hence high level of suspicion is required for timely diagnosis of this febrile illness.

Eschar has been considered pathognomic of scrub typhus infection, though compared to global literature, India reports fewer number of patients (8-15\%) with eschar formation $[6,9,17]$. The cause can be mostly attributed to the dark complexion of the inhabitants and lower degree of suspicion of the disease among the physicians. One study from South India have reported presence of eschar in $43.5 \%$ of their study population [15]. They are mostly found in areas of moisture and tight clothing, like in axilla, breast and groins. A detailed clinical examination of the generalized skin condition should be carried out in these cases and absence of eschar does not rule out the diagnosis of scrub typhus in Indian setting.

The most common electrolyte abnormality in the study group was hyponatremia. The reason behind hyponatremia may be multiple including sodium loss, cellular sodium influx due to decreased sodium potassium ATP-ase activity, increased level of ADH (anti diuretic hormone) and osmo-receptor resetting. Various studies conducted all over India from different states also reveal similar biochemical spectrum as shown in the present study $[7,13,15,16]$.

Various studies have tried to bring forth a clinical score to differentiate scrub typhus and other undifferentiated febrile illnesses like dengue in order to facilitate a prompt diagnosis by primary care physicians who may not have immediate access to confirmatory tests $[18,19]$. In a study by Varghese et al. $(n=50)$, a combination of elevated transaminases, thrombocytopenia and leucocytosis displayed $80 \%$ specificity and positive predictive value for diagnosis of scrub typhus [18]. Another study concluded that serum aminotransferase elevation is an independent predictor in the diagnosis of scrub typhus, especially in suspected patients without eschar [20].
Scrub typhus can manifest with potentially life threatening complications. The present study emphasizes on the occurrence of complications in a signification proportion $(89.7 \%)$ of study cohort, most common being ARDS, followed by sepsis with MODS and AKI. Similar results were obtained from other studies in India $[7,15]$. Systemic complications develop after the first week of illness and are directly related to the blood load of Orientia tsutsugamushi [21]. Both agent and host factors are decisive for the occurrence of complications, Orientia has 20 antigenically distinct serotypes and some strains seem to have higher virulence. Host factors like extremes of age, co-morbidities on admission and delayed onset of treatment are deciding factors behind the occurrence of systemic complications [7]. Several studies reveal that patients with extremes of age have higher rates of severe complications, though did not have significant higher mortality rates $[22,23]$. Occurrence of systemic complications like shock requiring inotropes, CNS dysfunction, renal failure are independent predictors of mortality [15]. Prolongation of prothrombin time has also been shown to be an independent marker predicting complications for these patients. The presence of complications in such higher proportion in the present study population $(89.7 \%)$ can be attributed to the fact that only complicated cases reach the tertiary care centre in Haryana. The case fatality rate is $18 \%$ in this study corroborating with the wide range of case fatality rate for scrub typhus (5-30\%) reported from India $[15,18]$ and across the globe [10].

Renal involvement is a common manifestation in scrub typhus infection and literature shows abnormal urinalysis in majority of the patients like low grade albuminuria, microscopic hematuria and pyuria suggesting predominant tubulo-interstitial involvement. The pathophysiology of acute kidney injury in scrub typhus can be attributed to as a part of multi organ dysfunction syndrome due to overwhelming sepsis, decreased renal perfusion due to volume depletion and increased vascular permeability. Other probable mechanisms include acute tubular necrosis caused by direct tubular toxicity, interstitial nephritis and pigment nephropathy due to rhabdomyolysis and thrombotic microangiopathy secondary to DIC [24].

ARDS is another serious complication of scrub typhus and delay in use of appropriate antibiotics can lead to mortality rates up to $25 \%$. Scrub typhus infection causes direct endothelial cell invasion and marked iNOS expression in the alveolar cells. Microscopic examination reveals diffuse alveolar damage with hyaline membrane formation and interstitial pneumonitis with infiltration of inflammatory cells. Hence any case of scrub typhus presenting with cough, dyspnea, raised white blood cell counts, increased hematocrit or total bilirubin are at increased risk to develop ARDS [2].

Another interesting observation is the involvement of the central nervous system in the studied patients. 
Meningitis although less defined in the literature has been observed in nearly one-sixth $(15.3 \%)$ of the patients included in the present study with cerebrospinal fluid (CSF) examination revealing lymphocytic pleocytosis and increased proteins. In a study differentiating scrub typhus meningitis from tubercular meningitis among 57 patients by Valappil et al, it has been shown that lesser duration of symptoms, lower magnitude of neurological deficits, motor and cranial nerve deficits, lower CSF - WBC count, lower CSF protein elevation and increased CSF sugar are predictors of scrub typhus meningitis [25]. Moreover, increased serum glutamate oxaloacetate transaminase levels more than $40 \mathrm{U} / \mathrm{L}$, increased serum glutamate pyruvate transaminase levels more than $60 \mathrm{U} / \mathrm{L}$ are indicative of scrub typhus meningitis. Features differentiating scrub typhus meningitis from bacterial meningitis, as shown by Varghese et al, in a study on 25 patients, are duration of fever at presentation more than 5 days, CSF WBC of a lesser magnitude, CSF lymphocytosis more than $50 \%$ and serum ALT elevation more than $60 \mathrm{IU} / \mathrm{L}$ [26].

Approximately $22.7 \%$ of patients with scrub typhus present with gastrointestinal manifestations, predominantly abdominal pain, nausea, vomiting, hematemesis and diarrhea [28]. Gastric mucosal erosions and ulcerations owing to focal and disseminated vasculitis, resulting in gastrointestinal bleed is common in scrub infection. This may worsen a pre-existing peptic ulcer, causing duodenal perforation, and present as acute abdomen $[27,28]$.

One patient of scrub typhus developed acute myocarditis as evident by nonspecific ST-T changes in ECG, hemodynamic compromise, and left ventricular dysfunction in echocardiogram. Patient recovered completely after few days of inotropic support and prompt institution of anti-rickettsial treatment.

In the present study, $82 \%$ of patients responded to doxycycline administered $200 \mathrm{mg} /$ day in two divided doses for 14 days. A meta- analysis by Panpanich et al, reveals the paucity of high quality evidence on the antibiotics for scrub typhus [29]. However, existing evidences denote comparable efficacy between doxycycline, telithromycin, azithromycin and use of rifampicin in case of poor response to standard anti-rickettsial treatment.

\section{Conclusion}

Increased outdoor activities, lack of personal protective measures increase the chances of infection with vector borne endemic diseases like scrub typhus, malaria, leptospirosis and dengue in India. Since clinical spectrum often overlaps, diagnosis becomes a challenge. This study emphasizes that scrub typhus infection is on a rampant resurgence and associated with significant complications which can result in increased morbidity and mortality. High degree of suspicion as well as concurrent confirmatory testing of the above mentioned more prevalent diseases are crucial to avert the complications and mortality.

\section{References}

1. Aggarwal HK, Jain D, Kaverappa V, Mittal A, Yadav S, Gupta A. Emergence of Scrub Typhus in Northern India: Experience from Tertiary Care Hospital. Klimik Dergisi. 2014;27:6-11.

2. Wang CC, Liu SF, Liu JW, Chung YH, Su MC, Lin MC. Acute respiratory distress syndrome in scrub typhus. Am J Trop Med Hyg. 2007;76:1148-1152.

3. Yen TH, Chang CT, Lin JL, Jiang JR, Lee KF. Scrub typhus: a frequently overlooked cause of acute renal failure. Ren Fail. 2003;25:397-410.

4. Cracco C, Delafosse C, Baril L, Lefort Y, Morelot C, Derenne JP, et al. Multiple organ failure complicating probable scrub typhus. Clin Infect Dis. 2000;31:191-192.

5. Thap LC, Supanaranond W, Treeprasertsuk S, Kitvatanachai S, Chinprasatsak S, Phonrat B. Septic shock secondary to scrub typhus: characteristics and complications. Southeast Asian J Trop Med Public Health. 2002;33:780-786.

6. Masand R, Yadav R, Purohit A, Tomar BS. Scrub typhus in rural Rajasthan and a review of other Indian studies. Paediatr Int Child Health. 2016;36:148-153.

7. Sivarajan S, Shivalli S, Bhuyan D, Mawlong M, Barman R. Clinical and paraclinical profile, and predictors of outcome in 90 cases of scrub typhus, Meghalaya, India. Infect Dis Poverty. 2016;5:91.

8. Walker DH, Dumler S, Marrie T. 211: Rickettsial Diseases. In: Longo DL, Kasper DL, Jameson JL, Fauci AS, Hauser SL, Loscalzo J, et al., (eds.). Harrison's principles of internal medicine. 19th ed. New York:McGraw Hill; 2015, pp: 1154-1162. 9. Bhargava A, Kaushik R, Kaushik RM, Sharma A, Ahmad S, Dhar M, et al. Scrub typhus in Uttarakhand \& adjoining Uttar Pradesh: Seasonality, clinical presentations \& predictors of mortality. Indian J Med Res. 2016;144:901-909.

10. Bonell A, Lubell Y, Newton PN, Crump JA, Paris DH. Estimating the burden of scrub typhus: A systematic review. PLoS Negl Trop Dis. 2017;11:e0005838.

11. Isaac R, Varghese GM, Mathai E, J M, Joseph I. Scrub typhus: prevalence and diagnostic issues in rural Southern India. Clin Infect Dis. 2004;39:1395-1396.

12. Jung HC, Chon SB, Oh WS, Lee DH, Lee HJ. Etiologies of acute undifferentiated fever and clinical prediction of scrub typhus in a non-tropical endemic area. Am J Trop Med Hyg. 2015;92:256-261.

13. Rawat V, Singh RK, Kumar A, Saxena SR, Varshney U, Kumar M. Epidemiological, clinical and laboratory profile of scrub typhus cases detected by serology and RT-PCR in Kumaon, Uttarakhand: a hospital-based study. Trop Doct. 2018;48:103106.

14. Takhar RP, Bunkar ML, Arya S, Mirdha N, Mohd A. Scrub typhus: A prospective, observational study during an outbreak in Rajasthan, India. Natl Med J India. 2017;30:69-72.

15. Varghese GM, Trowbridge P, Janardhanan J, Thomas K, Peter JV, Mathews P, et al. Clinical profile and improving mortality trend of scrub typhus in South India. Int J Infect Dis. 2014;23:3943.

16. Narayanasamy DK, Arunagirinathan AK, Kumar RK, Raghavendran VD. Clinico-Laboratory Profile of Scrub Typhus - An Emerging Rickettsiosis in India. Indian J Pediatr. 2016;83:1392-1397.

17. Kularatne SA, Edirisingha JS, Gawarammana IB, Urakami H, Chenchittikul M, Kaiho I. Emerging rickettsial infections in Sri Lanka: the pattern in the hilly Central Province. Trop Med Int 
Health. 2003;8:803-811.

18. Varghese GM, Abraham OC, Mathai D, Thomas K, Aaron R, Kavitha ML, et al. Scrub typhus among hospitalised patients with febrile illness in South India: magnitude and clinical predictors. J Infect. 2006;52:56-60.

19. Mitra S, Gautam I, Jambugulam M, Abhilash KP, Jayaseeelan V. Clinical Score to Differentiate Scrub Typhus and Dengue: A Tool to Differentiate Scrub Typhus and Dengue. J Glob Infect Dis. 2017;9:12-17.

20. Su TH, Liu CJ, Shu PY, Fu YH, Chang CH, Jao P, et al. Associated factors and clinical implications of serum aminotransferase elevation in scrub typhus. J Microbiol Immunol Infect. 2016;49:941-946.

21. Sonthayanon P, Chierakul W, Wuthiekanun V, Phimda K, Pukrittayakamee S, Day NP, et al. Association of high Orientia tsutsugamushi DNA loads with disease of greater severity in adults with scrub typhus. J Clin Microbiol. 2009;47:430-434.

22. Chang K, Lee NY, Ko WC, Lin WR, Chen YH, Tsai JJ, et al. Characteristics of scrub typhus, murine typhus, and $\mathrm{Q}$ fever among elderly patients: Prolonged prothrombin time as a predictor for severity. J Microbiol Immunol Infect. 2017;S16841182(17)30066-X.

23. Kalal BS, Puranik P, Nagaraj S, Rego S, Shet A. Scrub typhus and spotted fever among hospitalised children in South India:
Clinical profile and serological epidemiology. Indian J Med Microbiol. 2016;34:293-298.

24. Kumar V, Kumar V, Yadav AK, Iyengar S, Bhalla A, Sharma $\mathrm{N}$, et al. Scrub typhus is an under-recognized cause of acute febrile illness with acute kidney injury in India. PloS Negl Trop Dis. 2014;8:e2605.

25. Valappil AV, Thiruvoth S, Peedikayil JM, Raghunath $P$, Thekkedath M. Differential diagnosis of scrub typhus meningitis from tuberculous meningitis using clinical and laboratory features. Clin Neurol Neurosurg. 2017;163:76-80.

26. Varghese GM, Mathew A, Kumar S, Abraham OC, Trowbridge P, Mathai E. Differential diagnosis of scrub typhus meningitis from bacterial meningitis using clinical and laboratory features. Neurol India. 2013;61:17-20.

27. Lee CH, Lee JH, Yoon KJ, Hwang JH, Lee CS. Peritonitis in patients with scrub typhus. Am J Trop Med Hyg. 2012;86:10461048.

28. Rajat R, Deepu D, Jonathan AJ, Prabhakar AK. Duodenal Perforation Precipitated by Scrub Typhus. J Glob Infect Dis. 2015;7:87-88.

29. Kim SJ, Chung IK, Chung IS, Song DH, Park SH, Kim HS, et al. The clinical significance of upper gastrointestinal endoscopy in gastrointestinal vasculitis related to scrub typhus. Endoscopy. 2000;32:950-955. 